\section{Bilateral adrenal masses: phaeochromocytoma or melanoma?}

\author{
Isla S Mackenzie1 Michael J Ashby² \\ Tim Donovan ${ }^{4}$ Demetrius D Voutnis ${ }^{5}$ \\ Morris J Brown ${ }^{3}$
}

J R Soc Med 2006;99:153-155

Adrenal masses vary in importance from being incidental findings of no clinical significance to having sinister consequences through malignancy. The importance in integrating the clinical history, biochemical findings and results of modern imaging techniques is illustrated in this case.

\section{CASE HISTORY}

A man of 70 presented to his general practitioner with a 2 month history of weight loss, anorexia, nausea and abdominal pains. Past medical history included pharyngeal pouch and excision of a malignant melanoma from his left shoulder 7 years previously. The previous melanoma was a $1 \mathrm{~cm}$ maximum diameter superficial spreading type of malignant melanoma showing invasion of the epidermis and superficial dermis with a maximum depth of $0.6 \mathrm{~mm}$. The resection margins were clear. The patient had shown no clinical evidence of local recurrence or axillary lymphadenopathy at 6-monthly reviews and was discharged after 5 years of follow-up according to the prevalent protocol.

Physical examination was unremarkable except for a scar on his shoulder. Routine blood tests and chest radiograph were normal. He was referred to his local hospital where he was seen a month later and was found to be hypertensive, with a blood pressure of 190/96 $\mathrm{mmHg}$, for which he was commenced on lisinopril. Abdominal ultrasound and subsequent computerized tomography scanning (Figure 1) revealed enlargement of both adrenal glands, reported as 'probably benign'. In view of the hypertension and adrenal masses, urinary catecholamines were measured. 24-hour urinary noradrenaline (254 nmol/24 h) and adrenaline (21 $\mathrm{nmol} / 24 \mathrm{~h}$ ) excretion were normal, but 24-hour urinary dopamine was grossly elevated at $18783 \mathrm{nmol}$ (normal $<3194 \mathrm{nmol}$ ). He was referred to the tertiary centre (3

${ }^{1}$ Clinical Lecturer, ${ }^{2}$ Research Associate, ${ }^{3}$ Professor of Clinical Pharmacology, Clinical Pharmacology Unit, ${ }^{3}$ Senior Radiographer, Wolfson Brain Imaging Centre; ${ }^{4}$ Specialist Registrar, Department of Nuclear Medicine, Addenbrooke's Hospital, Cambridge CB2 2QQ, UK

Correspondence to: Dr Isla S Mackenzie

E-mail: ism22@cam.ac.uk

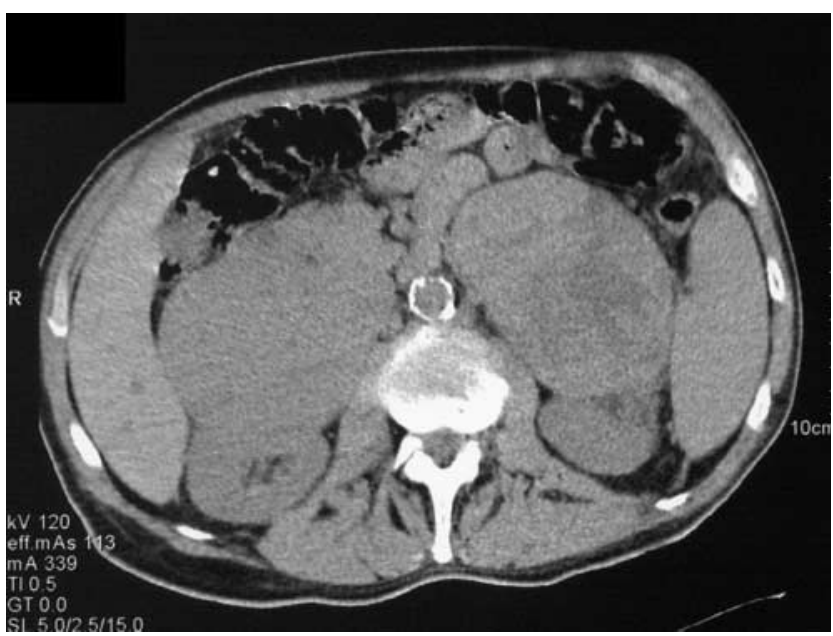

Figure 1 Abdominal CT scan showing bilateral adrenal masses

months after his initial presentation to his general practitioner) for further investigation of possible bilateral dopamine secreting phaeochromocytoma.

He was seen the next day. By now he was generally unwell with nausea and vomiting, and had lost $20 \mathrm{~kg}$ in weight since the start of his illness. He had no palpitations, sweats or fainting. He looked cachectic and tanned. There was no palpable lymphadenopathy. Blood pressure was 159/85 mmHg. Large masses were palpable in both abdominal flanks. Adrenal insufficiency was suspected and after performance of a short Synacthen test (baseline cortisol $296 \mathrm{nmol} / \mathrm{L}$, 30-min cortisol $268 \mathrm{nmol} / \mathrm{L}$ ) he was started on hydrocortisone. His symptoms improved dramatically with increases in appetite and weight, and rapid resolution of the nausea and vomiting.

After correction of his hypoadrenalism, routine blood tests were normal. Plasma noradrenaline $(2.79 \mathrm{nmol} / \mathrm{L}$; normal range $0.59-5.33 \mathrm{nmol} / \mathrm{L})$ and adrenaline $(<0.055$ $\mathrm{nmol} / \mathrm{L}$; normal range $<0.82 \mathrm{nmol} / \mathrm{L}$ ) were not elevated,

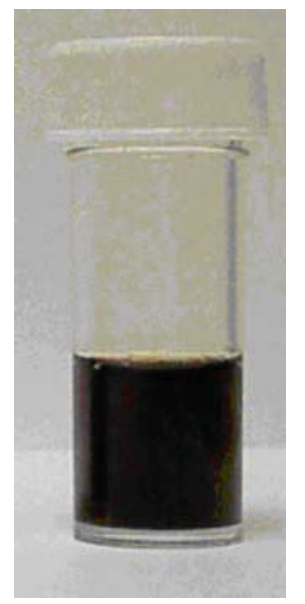

Figure 2 Melanuria-a specimen of the patient's urine that had been left standing. The dark colour is due to the oxidation of melanogens in the urine. 

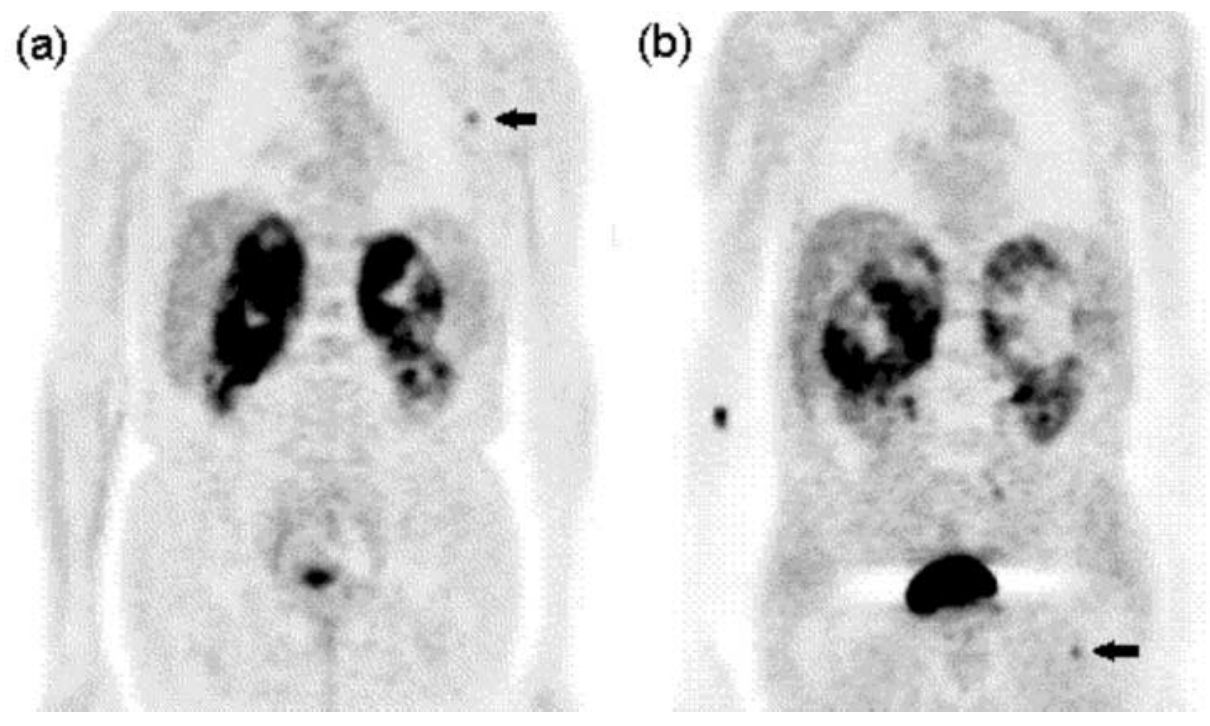

Figure 3 (a) ${ }^{18} \mathrm{~F}$-fluorodeoxyglucose (FDG) PET scan showing abnormal uptake of tracer in the adrenal masses and left axilla (arrow). (b) 6-[ ${ }^{18}$ F]-fluoro-L-DOPA (F-DOPA) PET scan showing abnormal uptake of tracer in the adrenal masses and left inguinal region (arrow).

and plasma dopamine only mildly so $(0.44 \mathrm{nmol} / \mathrm{L}$; normal range $<0.33 \mathrm{nmol} / \mathrm{L})$. In contrast, a urine sample again showed a very high dopamine level of 30,669 nmol/L. Interestingly, it was noticed that a specimen of his urine turned dark when left standing overnight (Figure 2). A MIBG ( ${ }^{123}$ I-metaiodobenzylguanidine) scan to exclude phaeochromocytoma showed physiological uptake of isotope only.

In order to assess whether the adrenal masses were metastases from his previous melanoma, we performed an ${ }^{18}$ F-fluorodeoxyglucose (FDG) positron emission tomography (PET) scan, which highlighted both adrenal masses and a small area of abnormal uptake in the left axilla (Figure $3 a$ ). Similar adrenal uptake was seen on a further scan using 6 - $\left[{ }^{18} \mathrm{~F}\right]$-fluoro-L-DOPA (dihdroxyphenylalanine), as well as an area in the left inguinal region (Figure 3b), consistent with our suspicion that the patient had metastatic melanoma.

Although curative resection for isolated adrenal metastases is well described, ${ }^{1,2}$ this option was excluded by the discovery of other metastases. Repeat computerized tomography scanning confirmed enlarged left axillary lymph nodes together with further enlargement of the adrenals and loss of clear tissue planes.

Despite replacement steroids and a course of palliative dacarbazine chemotherapy, the patient died 9 months after first detection of the adrenal masses.

\section{DISCUSSION}

This case illustrates the potential for diagnostic confusion between dopamine-secreting phaeochromocytoma and metastatic malignant melanoma and demonstrates how facilitates correct diagnosis (see below). The very late presentation of clinically significant hypoadrenalism several years after the excision of a primary low-grade malignant melanoma is unusual. Other interesting features of the case include the use of FDG-PET scanning and the less common technique of F-DOPA PET scanning to help to confirm the diagnosis and avoid the need for biopsy.

\section{Explanation for elevated urinary dopamine}

Adrenal masses associated with several-fold elevation of urinary dopamine may at first sight seem diagnostic of dopamine-secreting phaeochromocytoma. However, pure dopamine secreting phaeochromocytomas in adults are very rare and tend to present with symptoms of hypotension, vomiting, diarrhoea and collapse. Plasma dopamine levels are typically several-fold elevated. This patient's plasma dopamine was only slightly elevated while, in contrast, the urinary dopamine levels were extremely high. DOPA is an intermediary in the synthesis of melanin from tyrosine, with the same enzyme - tyrosine hydroxylase - catalysing both the initial synthesis of DOPA and its subsequent conversion to dopaquinone, the precursor of the melanins (Figure 4). Induction of tyrosine hydroxylase in melanoma tissue leads to the formation of excessive amounts of DOPA, melanogens and melanins. High circulating levels of DOPA are converted to dopamine by the enzyme DOPA decarboxylase during renal excretion. DOPA decarboxylase is mainly located in the kidneys, which explains the greatly elevated dopamine levels found in this patient's urine. ${ }^{3}$

\section{Melanuria}

The darkening of the urine on standing was probably due to oxidation of melanogens. Although the intermediary 


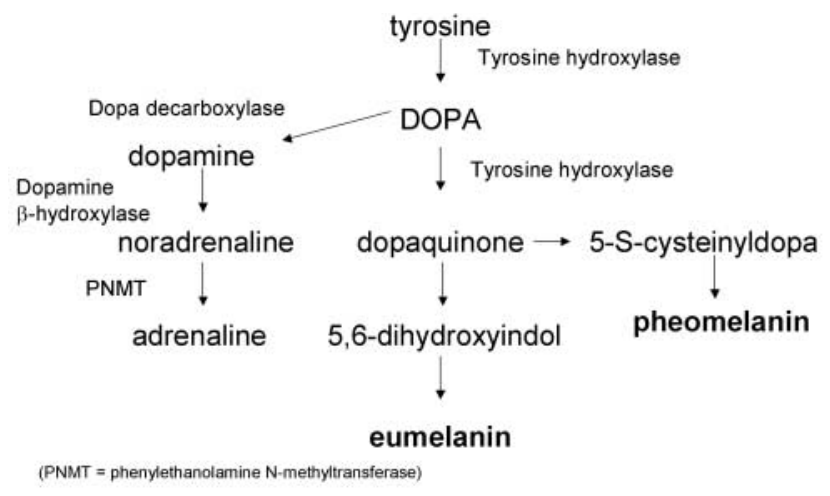

Figure 4 Melanin and catecholamine biosynthetic pathways. Induction of tyrosine hydroxylase in melanoma tissue leads to the formation of excessive amounts of DOPA, melanogens and melanins. High circulating levels of DOPA are converted to dopamine by the enzyme DOPA decarboxylase (which is mainly present in the kidney) during renal excretion, causing greatly elevated dopamine levels in the urine.

metabolism of melanin is well established, the high urinary dopamine and melanuria in malignant melanoma have not been the focus of intensive investigation. Unfortunately, neither elevated urinary dopamine nor melanuria are considered sufficiently sensitive or specific to form a useful diagnostic test in malignant melanoma. ${ }^{4,5}$ However, an understanding of the link between the pathways for catecholamine metabolism and melanogenesis was a major indicator of the likely diagnosis in this case.

\section{PET scanning in the diagnosis of adrenal masses}

Adrenal masses often present a diagnostic problem. Fine needle biopsy may be useful, but is not recommended for possible phaeochromocytoma. PET scanning complements computerized tomography and magnetic resonance scanning by providing more functional information about the adrenal masses. FDG-PET scanning is frequently used to locate tumours or metastases in patients with known primaries, including melanomas. F-DOPA PET scanning has mainly been used as a research tool in studies of the brain, and more recently in the imaging of phaeochromocytoma, and is less widely available than FDG. Whereas any metabolically active tissue will accumulate FDG, a follow-up scan with FDOPA, as in our patient, may help confirm the origin of FDG hotspots and increases sensitivity for detecting metastases in melanoma. ${ }^{6}$

\section{Hypoadrenalism and metastatic malignant melanoma}

Adrenal metastases may present several years after the initial presentation of melanoma and usually occur in patients in whom the original lesion was judged as locally advanced. However, the only previous reports of such patients developing clinically significant hypoadrenalism have been at or shortly after initial presentation of the melanoma $^{2}$. Over $90 \%$ of the adrenal cortices must be destroyed before there is significant loss of function. Adrenal dysfunction may either develop gradually due to replacement of adrenal tissue with tumour, or suddenly due to acute adrenal haemorrhage. It has been suggested that prophylactic steroids should be given to any patient with known adrenal metastases to avoid the risk of hypoadrenal crisis due to adrenal haemorrhage. ${ }^{7}$ There are reports of successful outcomes following adrenalectomy for patients with metastatic melanoma isolated to the adrenal glands. ${ }^{1,2}$ However, the prognosis is significantly poorer if only incomplete resection of disease can be achieved. ${ }^{8}$

In summary, this case of a patient with malignant melanoma with hypoadrenalism and excess urinary dopamine excretion illustrates the importance of asking about any history of tumours when diagnosing adrenal masses, and highlights the value of integrating the history into the diagnostic process and of linking clinical features with knowledge of biochemical pathways.

Acknowledgments PET scanning at Addenbrooke's Hospital is supported by a Medical Research Council Wolfson Brain Imaging Centre Cooperative Group Grant. IM is supported by a British Heart Foundation Clnical PhD Studentship and a Sackler Award.

Competing interests None declared.

\section{REFERENCES}

1 Haigh PI, Essner R, Wardlaw JC, Stern SL, Morton DL. Long-term survival after complete resection of melanoma metastatic to the adrenal gland. Ann Surg Oncol 1999;6:633-9

2 Lam KY, Lo CY. Metastatic tumours of the adrenal glands: a 30-year experience in a teaching hospital. Clin Endocrinol (Oxf) 2002;56:95-101

3 Brown MJ, Dollery CT. A specific radioenzymatic assay for dihydroxyphenylalanine (DOPA). Plasma dopa may be the precursor of urine free dopamine. Br J Clin Pharmacol 1981;11:79-83

4 Scott RE, Wilson DM. Role of the clinical laboratory in the diagnosis and management of malignant melanoma. Mayo Clin Proc 1989; 64:83745

5 Faraj BA, Lawson DH, Nixon DW, et al. Melanoma detection by enzyme-radioimmunoassay of L-dopa, dopamine, and 3-Omethyldopamine in urine. Clin Chem 1981;27:108-12

6 Dimitrakopoulou-Strauss A, Strauss LG, Burger C. Quantitative PET studies in pretreated melanoma patients: a comparison of 6-[18F]fluoroL-dopa with 18F-FDG and (15)O-water using compartment and noncompartment analysis. J Nucl Med 2001;42:248-56

7 Das Gupta T, Brasfield R. Metastatic melanoma. A clinicopathological study. Cancer 1964;17:1323-39

8 Wood TF, DiFronzo LA, Rose DM, Haigh PI, Stern SL, Wanek L et al. Does complete resection of melanoma metastatic to solid intraabdominal organs improve survival? Ann Surg Oncol. 2001;8:658-62 